\title{
Prevalence of vulvovaginitis and relation to physical findings in girls assessed for suspected child sexual abuse
}

\author{
Gisel Rahman,M.D. ${ }^{a}$, Dolores Ocampo,M.D. ${ }^{a}$,AnahíRubinstein, M.D. ${ }^{a}$, and Paula Risso, B.S. ${ }^{b}$
}

\begin{abstract}
Introduction. The presence of sexually transmitted infections (STIs) in patients with suspected sexual abuse is uncommon in the field of pediatrics.

Objectives. To establish the prevalence of anogenital findings and their relation to the presence of STIs in girls referred for suspected child sexual abuse.

Material and Methods. Retrospective study conducted between January 1 $1^{\text {st }}, 2003$ and December 31 ${ }^{\text {st }}, 2013$. Physical findings and detection of STIs in girls with suspected child sexual abuse were analyzed.

Results. One thousand thirty-four patients were included. Their median age was 7.9 years old. Anogenital findings were classified as class I (normal): $38.4 \%$, class II (nonspecific): $38.1 \%$, class III (specific): $19.9 \%$ and class IV (definitive): $3.6 \%$. STIs were observed in 42 patients $(4.1 \%)$. A relation was established between STIs and the classification of physical findings: 10 (class II: 9; class III: 1) Neisseria gonorrhoeae, 17 (class I: 2; class II: 8; class III: 7) Chlamydia trachomatis, 15 (class I: 2; class II: 10; class III: 3) Trichomonas vaginalis. Statistically significant differences for Trichomonas vaginalis $(p=0.01)$ and Neisseria gonorrhoeae $(p<$ 0.0001 ) were observed, with predominance of nonspecific clinical signs. Both nonspecific and specific findings were similarly observed for Chlamydia trachomatis ( $p=0.03$ ).

Conclusions. Most cases of girls with suspected child sexual abuse had normal or nonspecific anogenital findings. The prevalence of STIs in these girls is low. Trichomonas vaginalis and Neisseria gonorrhoeae were related to nonspecific findings, while both nonspecific and specific findings were observed for Chlamydia trachomatis. Key words: physical exam, sexually transmitted infections, child sexual abuse, child, female.
\end{abstract}

http:/ /dx.doi.org/10.5546/aap.2015.eng.390

\section{INTRODUCTION}

Few areas of pediatrics have expanded so rapidly in clinical importance in recent years as that of child sexual abuse (CSA). ${ }^{1}$

Genital examination is considered a major element when examining a girl victim of abuse. Standards for terminology and classification schemes of physical findings have been established in several publications for the purpose of establishing a relation to the diagnosis of $\mathrm{CSA}^{2-4}$ and have become a useful diagnostic tool in clinical practice. However, several studies have demonstrated that diagnosis is not commonly based on physical findings. ${ }^{2,5,6}$ An absence of physical signs does not undermine the diagnosis of abuse, for which the girl's account and description are fundamental. ${ }^{2,7}$

The presence of sexually transmitted infections (STIs) in patients assessed for suspected sexual abuse is uncommon in the field of pediatrics. According to the Committee on Infectious Diseases of the American Academy of Pediatrics, it may reach $8.2 \%{ }^{8}$

\section{OBJECTIVES}

To establish the prevalence of anogenital findings and their relation to the presence of STIs in girls referred for suspected CSA to the Unit of Pediatric and Adolescent Gynecology of Hospital de Niños "Sor María Ludovica" in the city of La Plata (Buenos Aires, Argentina).

\section{MATERIAL AND METHODS Retrospective study}

Patients referred to the Unit of Pediatric and Adolescent Gynecology of Hospital de Niños "Sor María Ludovica" in La Plata for suspected CSA between January $1^{\text {st }}, 2003$ and December 31 ${ }^{\text {st }}, 2013$ were included. Patients were referred based on the girl's account, suspicion on part of a family member, a health care provider, or justice agencies, or detection of sexually-transmitted microorganisms. Case history, general 
physical exam and gynecological assessment were conducted in the presence of an adult caregiver. Anogenital findings were categorized based on the guidelines published by Muram\& Adams (see Annex I in electronic format). ${ }^{9}$

A vaginal discharge specimen was collected for vaginal swab from those patients who, at the time of the assessment, were at high risk for STIs due to the following situations: vaginal or anal penetration, contact with secretions, signs and symptoms suggestive of STI, presence of class III physical findings, a perpetrator with risk factors for an STI, a family member living with an STI, diagnosis of an STI in the girl. ${ }^{1,10} \mathrm{~A}$ fresh smear, Gram staining, culture for common microorganisms, direct immunofluorescence for Ureaplasma urealyticum and polymerase chain reaction (PCR) for Chlamydia trachomatis were done. Cultures were performed in chocolate agar and Thayer-Martin agar, in a wet environment with $5 \% \mathrm{CO}_{2}$ at a constant temperature of $37^{\circ} \mathrm{C}$. Suspicious colonies for Neisseria gonorrhoeae were identified using oxidase, superoxol and sugar utilization tests (lactose, sucrose, dextrose and maltose). Strains identified as Neisseria gonorrhoeae were referred to the National Administration of Health Institutes and Labs (ANLIS) "Dr. Carlos G. Malbrán" for confirmation. Positive cultures for Neisseria gonorrhoeae, Chlamydia trachomatis and Trichomonas vaginalis were considered markers of STIs (see Annex II in electronic version).

\section{Statistical method}

The proportion of microorganisms was compared with the different classes of physical findings using a Bayesian "comparison of a difference in proportions" methodology. ${ }^{11} \mathrm{~A}$ statistical significance level was established at $\alpha \leq 0.05$. Excel ${ }^{\circledR}$ was the software used as database, while Epidat $4.1^{\circledR}$ was used for statistical analysis. ${ }^{12}$

\section{Ethical considerations}

This was a retrospective study, so it was not possible to obtain an informed consent. Data were anonymized. The study was approved by the Teaching and Research Board of Hospital "Sor María Ludovica".

\section{RESULTS}

One thousand thirty-four patients were assessed. Their median age was 7.9 years old, ranging from 0.3 to 16.8 years old.

Findings corresponded to class I in $38.4 \%$ of patients $(\mathrm{n}=397)$; class II, in $38.1 \%(\mathrm{n}=394)$; class III, in $19.9 \%(n=206)$; and class IV, in 3.6\% $(n=37)$.

Criteria for vaginal swab collection for culture were recorded in $40.4 \%$ of cases $(418 / 1034)$, and $120 / 418$ resulted positive $(28.7 \%)$. STIs were recorded in $35 \%(42 / 120)$ of all patients with positive cultures, which accounts for $4.1 \%$ (42/1034) of all assessed patients. All infectious agents are described in Table 1.

The 37 cases included in class IV were not established by anogenital findings. They corresponded to different diagnoses: pregnancy $(n=10)$, syphilis $(n=9)$, positive human immunodeficiency virus (HIV) $(n=2)$, presence of sperm in urine $(n=2)$, witnesses to CSA $(n=3)$, perpetrator's confession $(n=1)$, and positive culture for gonococcus $(n=10)$. All patients in this category had no evidence of definitive anogenital findings (signs of penetrating trauma); observations corresponded to nonspecific and specific findings.

STI agents were related to the type of physical findings: genital examination was normal in 15 patients $(12.5 \%)$, nonspecific findings were observed in $61(50.8 \%)$, and specific findings, in 44 $(36.7 \%)$. Statistically significant differences were found between classes of findings for Chlamydia trachomatis $(p=0.03)$, Trichomonas vaginalis $(p=0.01)$ and Neisseria gonorrhoeae $(p<0.0001)$. Trichomonas vaginalis and Neisseria gonorrhoeae showed a significant predominance of nonspecific clinical signs when compared to normal findings and specific findings. In these patients, genital secretions, vulvar congestion and incomplete notch were observed. Chlamydia trachomatis was

TABLE 1. Frequency of infectious agents studied in the population with cultures (418 cases)

\begin{tabular}{lcc}
\hline Infectious agent & Cases & Percentage \\
\hline Positive cultures & $120 / 418$ & 28.7 \\
Gardnerella vaginalis & 38 & 9.1 \\
Ureaplasma urealyticum & 30 & 7.2 \\
Candida sp. & 20 & 4.8 \\
Haemophilus influenzae & 9 & 2.2 \\
Shigella sp. & 2 & 0.5 \\
Mycoplasma sp. & 1 & 0.2 \\
Chlamydia trachomatis & 17 & 4.1 \\
Trichomonas vaginalis & 15 & 3.6 \\
Neisseria gonorrhoeae & 10 & 2.4 \\
\hline
\end{tabular}

Microorganisms considered sexually transmitted infections are indicated in bold. 
significantly more common with both nonspecific and specific findings. These girls had a notch extending to the base of the hymen, enlarged hymenal orifice, and vulvar bruising (Table 2).

Gardnerella vaginalis was assessed in relation to the three classes of findings, with statistically significant differences observed $(p=0.005)$. Nonspecific and specific findings were significantly more common (Table 2).

\section{DISCUSSION}

Child sexual abuse is defined as sexual contacts forced by an adult on a child who, given his/her condition, lacks emotional and cognitive development to give his/her consent regarding sexual contact. According to some authors, children's average age ranges between 8 and 12 years old. ${ }^{2,13}$ The median age of our patients was 7.9 years old.

Sexual contact in pediatric patients generally does not involve vaginal and/or anal penetration; physical exam of girls victims of sexual abuse may show a normal hymen and no other physical findings. ${ }^{4,14}$ In our study, there was a low percentage of girls $(3.6 \%)$ with physical findings definitively suggestive of CSA, data which are consistent with other publications. ${ }^{2,6}$ A normal physical exam does not invalidate a diagnosis of abuse; the girl's account is fundamental.,

Approximately $5-8 \%$ of abused children get a sexually transmitted infection from their perpetrators. ${ }^{7,15,16}$ In our study, $4.1 \%$ of assessed patients $(42 / 1034)$ had an STI. Serological tests and cultures for STIs are not part of the routine for prepubertal girls. The account of the abuse, the presence of trauma signs, an infection or knowing that the perpetrator has an STI may help to establish what specific tests should be requested. The incidence of asymptomatic infections among adolescent girls is significant, and given the high prevalence of STI, this population should be studied..$^{10}$ In this group, serological tests for syphilis and HIV and cultures for Neisseria gonorrhoeae and Chlamydia trachomatis should be done.

According to the Centers for Disease Control and Prevention (CDC), the presence of different STIs in the diagnostic framework of CSA may be considered inconclusive, suspicious or diagnostic (see Annex II in electronic format). In our study, most girls with sexually transmitted infections had normal or nonspecific physical findings, as indicated in other publications. ${ }^{2,16}$

Isolation of Neisseria gonorrhoeae in specific cultures confirms CSA. ${ }^{9}$ Some authors suggest direct contact as a potential for transmission (hands, fomites). ${ }^{17}$ In our study, this microorganism was isolated in $2.4 \%$ of cultures, which is consistent with Girardet, who recorded a $3.3 \%$ isolation in a population of 536 patients. ${ }^{16}$ In our study, most patients had nonspecific findings: genital secretions, vulvar congestion and incomplete notch. No definitive physical signs were observed. Reading, et al., referred an association between Neisseria gonorrhoeae and the presence of vulvar symptoms, mainly genital secretions. $^{18}$

Although the presence of Trichomonas vaginalis in cultures is highly suspicious, $66.7 \%$ of isolated cases had nonspecific findings: vaginal secretion and incomplete notch, which is consistent with other authors. ${ }^{6,16,19}$

The association between Chlamydia trachomatis and child sexual abuse is more complex than gonococcal infection. It is diagnostic, if vertical

TABLE 2. Anogenital findings and identification of infectious agents in specimens cultured $(n=418)$

\begin{tabular}{lcccc}
\hline Infectious agent & Class I (15) & Class II (61) & Class III (44) & Total \\
\hline Gardnerella vaginalis & 5 & $15^{*}$ & $18^{*}$ & 38 \\
Ureaplasma urealyticum & 3 & 13 & 14 & 30 \\
Candida sp. & 2 & 9 & 9 & 20 \\
Haemophilus influenzae & 2 & 6 & 1 & 9 \\
Shigella sp. & & 2 & 1 & 2 \\
Mycoplasma sp. & 2 & $8^{*}$ & $7^{*}$ & 1 \\
Chlamydia trachomatis & 2 & $10^{*}$ & 3 & 17 \\
Trichomonas vaginalis & & $9^{*}$ & 1 & 0.03 \\
Neisseria gonorrhoeae & & 0.01 & 10
\end{tabular}

Class IV is not included because there were no definitive anogenital findings. The number of microorganisms recorded may not match the total number of studied patients due to the fact that some patients had more than one positive diagnosis. Microorganisms considered sexually transmitted infections are indicated in bold. In the horizontal reading of sexually transmitted infections and Gardnerella vaginalis, proportions among the three classes were statistically compared. Significantly more frequent $(p \leq 0.05)$ classes of physical findings are indicated with an asterisk $(*)$. 
transmission has been ruled out. In our study, Chlamydia trachomatis was isolated in $4.1 \%$ of studied patients: $47.1 \%$ of cases were associated to nonspecific findings, and $41.2 \%$, to specific findings. Although percentages are lower in some published studies (Kelly, et al.: 1.2\%; and Kohlberger, et al.: $1.6 \%$ ), the introduction of new detection techniques, such as PCR, may facilitate its isolation..$^{6,20}$

Detection of Gardnerella vaginalis is considered inconclusive for the diagnosis of abuse.

It has been extensively described in post pubertal girls victims of sexual assault, with a rate between 5\% and $42 \% .{ }^{21}$ Data from prepubertal girls are scarce and controversial; its isolated presence does not define CSA. ${ }^{1}$ Gardner, et al. compared a population of abused and not abused girls and found that it was much more common in the latter group. ${ }^{22}$ In our study, Gardnerella vaginalis was isolated in $9.1 \%$ of patients. Specific or class III findings corresponded to $47 \%$ of cases (presence of complete notch and enlarged hymenal orifice diameter for age); most were post pubertal patients.

Finally, we estimate that, if child sexual abuse is suspected and nonspecific findings are observed in the physical exam, the recommendation is to perform a vaginal swab for STIs. The girl's account is of vital importance for the diagnosis of abuse. In the past years, better training has been evidenced in pediatric practice in relation to how these patients are treated, not just from a medical perspective but also regarding their psychosocial environment; therefore, an integral and interdisciplinary approach is fundamental. ${ }^{23}$

Among the strengths of the study, we could mention that it delves deep into the relationship between physical findings at the time of assessment and sexually transmitted infections, a topic that has been scarcely published in the literature, and the number of thoroughly studied patients, which allowed us to make statistically valid conclusions.

In this study, STIs not diagnosed by vaginal swab or their relation to the different Tanner stages were not assessed.

\section{CONCLUSION}

Most cases of girls referred due to CSA had normal or nonspecific anogenital findings. The prevalence of STIs in these girls is low. Trichomonasvaginalis and Neisseria gonorrhoeae were related to nonspecific findings, while both nonspecific and specific findings were observed for Chlamydia trachomatis.

\section{REFERENCES}

1. Kellogg N. The evaluation of sexual abuse in children. Pediatrics 2005;116(2):506-12.

2. Heger A, Ticson L, Velasquez O, Bernier R. Children referred for possible sexual abuse: medical findings in 2384 children. Child Abuse Negl 2002;26(6-7):645-59.

3. Adams JA. Evolution of a classification scale: medical evaluation of suspected child sexual abuse. Child Maltreat 2001;6(1):31-6.

4. Berenson $A B$, Chacko MR, Wiemann CM, Mishaw CO, et al. A case-control study of anatomic changes resulting from sexual abuse. Am J Obstet Gynecol 2000;182(4):820-31.

5. Kellogg ND, Menard SW, Santos A. Genital anatomy in pregnant adolescents: "normal" does not mean "nothing happened". Pediatrics 2004;113(1 Pt 1):e67-9.

6. Kelly P,KohJ, Thompson JM. Diagnostic findings in alleged sexual abuse: symptoms have no predictive value.J Paediatr Child Health 2006;42(3):112-7.

7. Ocampo D,Laperchia M, Borsa A, GiugnioS, etal.Hallazgos ginecológicos en pacientes con sospecha de abuso sexual en un hospital pediátrico. Rev Soc Argent Ginecol Infanto Juvenil 2006;13(3):159-63.

8. Jenny C,Crawford-JakubiaKJE. The evaluation of children in the primary care setting when sexual abuse is suspected. Pediatrics 2013;132(2):e558-67.

9. Adams JA, Harper K, Knudson S. A proposed system for the classification of anogenital findings in children with suspected sexual abuse. Adolesc Pediatr Gynecol 1992; 5(2): 73-5.

10. Workowski KA, Berman S. Sexually transmitted diseases treatment guidelines, 2010. MMWR Recomm Rep 2010;59(RR-12):1-110.

11. Christensen R, Johnson W, Branscum A,Hanson TE. Bayesian ideas and data analysis: an introduction for scientists and statisticians. Boca Raton: CRC Press; 2010.

12. Servizo de Epidemioloxía de la Dirección Xeral de Innovación e Xestión da Saúde Pública de la Consellería de Sanidade. EPIDAT: Análisis epidemiológico de datos. Versión 4.1 para Windows. Santiago de Compostela:Xunta de Galicia; 2014. [Accessed on: March 26, 2015]. Available at: http://www.sergas.es/MostrarContidos_N3_T01. aspx?IdPaxina $=62713$.

13. Hammerschlag MR, Guillén CD. Medical and legal implications of testing for sexually transmitted infections in children. Clin Microbiol Rev 2010;23(3):493-506.

14. Shrier LA. Infecciones de transmisión sexual: clamidia, gonorrea, enfermedad inflamatoria pélvica y sífilis. En: Emans SJ, Laufer MR, eds. Emans, Laufer, Goldstein. Ginecología en la infancia y la adolescencia.6. ${ }^{a}$ ed. Philadelphia: Lippincott Williams \& Wilkins; 2013.Págs.325-48.

15. Simmons KJ, Hicks DJ. Child sexual abuse examination: is there a need for routine screening for $\mathrm{N}$ gonorrhoeae and C trachomatis? J Pediatr Adolescent Gynecol 2005;18(5): 343-5.

16. Girardet RG, Lahoti S, Howard LA, Fajman NN, et al. Epidemiology of sexually transmitted infections in suspected child victims of sexual assault. Pediatrics 2009;124(1):79-86.

17. Daval-Cote M, Liberas S, Tristan A, Vandenesch F, et al. Vulvovaginite à gonocoque chez l'enfantprépubère: infection sexuellementtransmisibleou contamination accidentelle? Arch Pediatr 2013;20(1):37-40.

18. Reading R, Rogstad K, Hughes G, Debelle G. Gonorrhoea, chlamydia, syphilis and trichomonas in children under 13 years of age: national surveillance in the UK and Republic of Ireland. Arch Dis Child 2014;99(8):712-6. 
19. Ingram DL, Everett VD, Lyna PR, White ST, et al. Epidemiology of adult sexually transmitted disease agents in children being evaluated for sexual abuse. Pediatr Infect Dis J 1992;11(11):945-50.

20. Kohlberger P, Bancher-Todesca D. Bacterial colonization in suspected sexually abused children. J Pediatr Adolesc Gynecol 2007;20(5):289-92.

21. LederMR,EmansSJ.Abuso sexualenlaniña y la adolescente.
En: Emans SJ, Laufer MR, eds. Emans, Laufer, Goldstein. Ginecología en la infancia y la adolescencia. 6a ed. Philadelphia: Lippincott Williams \&Wilkins; 2013. Págs.523-38.

22. Gardner JJ. Comparison of the vaginal flora in sexually abused and nonabused girls. J Pediatr 1992;120(6):872-7.

23. Preer G, Sorrentino D, Newton AW. Child abuse pediatrics: prevention, evaluation, and treatment. Curr Opin Pediatr 2012;24(2):266-73. 


\section{Annex I}

\section{CLASSIFICATION OF ANOGENITAL FINDINGS IN GIRLS WITH SUSPECTED CHILD SEXUAL ABUSE}

\section{Class I: Normal genital examination.}

Class II: Nonspecific findings of sexual abuse. Physical signs that may have been caused by sexual abuse or other etiologies: vulvitis, scratching, increased vascularity of vaginal introitus, skin fissures or abrasions in the frenum of labia, coalescence of the labia minora, presence of vaginal discharge. Irregular hymenal rim, hymenal notch which does not extend the full width of the hymen (cleft or notch). In the anal region: presence of perianal erythema or hyperpigmentation, venous congestion, anal fissures, perianal skin tags, reduced perianal folds, anal dilation with stool within the rectal ampulla.

Class III: Specific findings of sexual abuse. Presence of one or more signs suggestive of sexual abuse: recent or scarred hymenal tears, enlarged hymenal orifice diameter for age, vaginal mucosa tear, bite marks or other trauma signs, such as lacerations or bruising in the vulva. Presence of sexually transmitted microorganisms. In the anal region: superficial or deep tears, scarring, anal sphincter laxity, presence of condylomata or other sexually transmitted microorganisms, anal dilation of more than $20 \mathrm{~mm}$ without stool within the rectal ampulla.

Class IV: Definitive findings of sexual abuse. Presence of sperm or seminal fluid in or on the girl's body. Pregnancy. Evidence of blunt or penetrating trauma, such as hymenal laceration to the base or "complete cleft". Positive culture for Neisseria gonorrhoeae. Positive serology for syphilis or HIV (if vertical transmission has been ruled out).Witnessed sexual abuse or video or photo documentation of child being abused. Confession of the alleged perpetrator regarding the actions described. 


\section{Annex II}

\section{IMPLICATIONS OF COMMONLY ENCOUNTERED SEXUALLY TRANSMITTED INFECTIONS FOR DIAGNOSIS AND REPORTING OF SEXUAL ABUSE AMONG INFANTS AND PREPUBERTAL CHILDREN}

\begin{tabular}{cc} 
Sexually transmitted infection confirmed & Evidence for sexual abuse \\
\hline Gonorrhea* $^{*}$ Syphilis* & Diagnostic \\
Human immunodeficiency virus & Diagnostic \\
Chlamydia trachomatis* & Diagnostic \\
Trichomonas vaginalis & Diagnostic \\
Condyloma acuminata (anogenital warts)* & Highly suspicious \\
Genital herpes (genital location)* & Suspicious \\
Bacterial vaginosis & Suspicious \\
\hline
\end{tabular}

* If not likely to be perinatally acquired and rare nonsexual, vertical transmission is excluded.

** If not likely to be acquired perinatally or through transfusion.

*** Should be confirmed through culture or nucleic acid amplification testing.

Data from the Centers for Disease Control and Prevention. Sexually Transmitted Diseases Treatment Guidelines. 2010. MMWR 2010;59[RR-12]:93 and Kellogg Pediatrics 2005;116:506-512. 